\title{
SUBDIRECT SUMS OF RINGS
}

\author{
NEAL H. MCCOY
}

1. Introduction. In much of the rather extensive theory of rings, the rings considered are restricted by certain finiteness assumptions. Under these assumptions the notion of direct sum plays an important role, but for general rings the usual theorems about direct sums are no longer valid. However, in certain important cases it turns out that the concept of subdirect sum is an appropriate generalization of that of direct sum. The purpose of this address is to summarize some of the more important known results concerning subdirect sums of rings. Following the introduction of the necessary definitions, we shall give an indication of the nature of these results.

2. Notation and preliminary concepts. Let $S_{i}(i=1,2, \cdots, n)$ be given rings, and denote by $S$ the set of all symbols

$$
\left(s_{1}, s_{2}, \cdots, s_{n}\right) \quad\left(s_{i} \in S_{i}, i=1,2, \cdots, n\right) .
$$

If we define addition and multiplication of these symbols by $\left(s_{1}, s_{2}, \cdots, s_{n}\right)+\left(s_{1}^{\prime}, s_{2}^{\prime}, \cdots, s_{n}^{\prime}\right)=\left(s_{1}+s_{1}^{\prime}, s_{2}+s_{2}^{\prime}, \cdots, s_{n}+s_{n}^{\prime}\right)$, and

$$
\left(s_{1}, s_{2}, \cdots, s_{n}\right)\left(s_{1}^{\prime}, s_{2}^{\prime}, \cdots, s_{n}^{\prime}\right)=\left(s_{1} s_{1}^{\prime}, s_{2} s_{2}^{\prime}, \cdots, s_{n} s_{n}^{\prime}\right),
$$

it is easy to verify that $S$ is a ring which we call the direct sum of the rings $S_{i}(i=1,2, \cdots, n)$. The set of all elements of $S$ of the form

$$
\left(s_{1}, 0, \cdots, 0\right)
$$

is clearly a subring $S_{1}^{\prime}$ of $S$ which is isomorphic to $S_{1}$ under the correspondence

$$
s_{1} \leftrightarrow\left(s_{1}, 0, \cdots, 0\right) .
$$

Furthermore, $S_{1}^{\prime}$ is closed under multiplication on either side by elements of $S$ and hence is not only a subring of $S$ but actually a twosided ideal in $S$. If, in like manner, we define $S_{i}^{\prime}(i=2,3, \cdots, n)$, it is clear that every element of $S$ is expressible uniquely in the form

$$
s_{1}^{\prime}+s_{2}^{\prime}+\cdots+s_{n}^{\prime} \quad\left(s_{i}^{\prime} \in S_{i}^{\prime}, i=1,2, \cdots, n\right),
$$

An address delivered before the New York meeting of the Society on February 22, 1947, by invitation of the Committee to Select Hour Speakers for Eastern Sectional Meetings; received by the editors March 12, 1947. 
the addition being ring addition in $S$. In the literature, $S$ is usually said to be the direct sum of its ideals $S_{i}^{\prime}$ and this is sometimes indicated by writing

$$
S=S_{1}^{\prime}+S_{2}^{\prime}+\cdots+S_{n}^{\prime} .
$$

However, for our purposes, it is usually more convenient to use the definition given above, that is, to consider $S$ as the direct sum of the rings $S_{i}$ which, although not subrings of $S$, are respectively isomorphic to the ideals $S_{i}^{\prime}$ in $S$. Obviously, the two concepts coincide up to an isomorphism.

The direct sum of the rings $S_{i}(i=1,2, \cdots, n)$ may be reformulated as the ring of all functions defined on a set of $n$ "points," say $\{1,2, \cdots, n\}$, such that at the point $i$ the functional values are in $S_{i}$. This immediately suggests the following generalization. Let $\mathfrak{A}$ be an arbitrary set of "points" and suppose that with each point $\alpha$ of $\mathfrak{A}$ there is associated a unique ring $S_{\alpha}$. The ring of all functions $f$ defined on $\mathfrak{A}$ such that $f(\alpha) \in S_{\alpha}(\alpha \in \mathfrak{U})$ is the direct sum of the rings $S_{\alpha}(\alpha \in \mathfrak{Y})$. In considering the direct sum of such a set of rings we shall find it convenient to use a notation and terminology which may suggest that the set $\mathfrak{A}$ is denumerable. However, we do not mean to imply any restriction on the cardinal number of the set $\mathfrak{A}$, or even that the set is necessarily well-ordered.

With this understanding, let $S_{i}(i=1,2, \cdots)$ be an arbitrary set of rings, distinct or identical, and let us denote an element $s$ of the direct sum $S$ of this set of rings by

$$
s=\left(s_{1}, s_{2}, \cdots\right) \quad\left(s_{i} \in S_{i}\right) .
$$

The zero of $S$ is clearly $(0,0, \cdots)$, the 0 in the $i$ th place being the zero of $S_{i}$. Now let $T$ be a subring of $S$. If

$$
t=\left(t_{1}, t_{2}, \cdots\right)
$$

is an arbitrary element of $T$, the correspondence

$$
t \rightarrow t_{i}
$$

defines a homomorphism of $T$ into $S_{i}$, in other words, a homomorphism of T onto a subring $S_{i}^{*}$ of $S_{i}$. The case of principal interest is that in which $S_{i}^{*}=S_{i}(i=1,2, \cdots)$, that is, under each homomorphism $t \rightarrow t_{i}$ every element of $S_{i}$ is the image of some element of $T$. If this is true we say that $T$ is a subdirect sum of the rings $S_{i}(i=1,2, \cdots)$, and that each $S_{i}$ is a component of $T$.

If the ring $R$ is isomorphic to a subdirect sum $T$ of rings $S_{i}(i=1$, $2, \cdots), T$ may be called a representation of $R$ as a subdirect sum of 
the rings $S_{i}(i=1,2, \cdots)$. If the isomorphism of $R$ and $T$ is given by

$$
r \leftrightarrow t,
$$

it is clear that the correspondence

$$
r \leftrightarrow t \rightarrow t_{i}
$$

defines a "natural" homomorphism of $R$ onto $S_{i}$.

Various problems now suggest themselves as follows. Given a ring $R$, find representations of $R$ as a subdirect sum. Do such representations always exist? Or, find necessary and sufficient conditions that a ring $R$ have a representation as a subdirect sum of rings of some specified kind. The main part of this address will be devoted to giving partial answers to certain specific questions of this general type.

The earliest references to infinite direct sums of rings or to subdirect sums of any type are Prüfer [24], ${ }^{1}$ Krull [14] and Köthe [11, 12]. Later on, we shall mention some of the essential contributions of these authors. However, much of the credit for the recent interest in this subject goes to Stone [26] for his fundamental work on the representations of Boolean rings. At this point it may be in order to quote one of Stone's results since it will illustrate the type of theorem to be expected.

A ring $R$ of more than one element is a Boolean ring if $a^{2}=a$ for every element $a$ of $R$. It is not difficult to show, either directly as in Stone [26] or as a special case of a well known general theorem, that a Boolean ring with a finite number of elements is isomorphic to a direct sum of a finite number of two-element fields, each of which we may consider to be the field $I /(2)$ of integers modulo 2 . For unrestricted Boolean rings this result does not carry over but instead we have the following result of Stone [26]:

Theorem 1. A ring $R$ is a Boolean ring if and only if it is isomorphic to a subdirect sum of fields $I /(2)$.

To show that the word "subdirect" in the statement of the theorem can not be replaced by "direct," it is only necessary to observe that the direct sum of any number of fields has a unit element but that there exist Boolean rings without unit element.

As a matter of fact, most of the known theorems about subdirect sum representations are generalizations of Theorem 1 in the sense that they reduce to this theorem in case the rings considered are specialized to be Boolean rings. An equivalent, and perhaps more

1 Numbers in brackets refer to the bibliography at the end of the paper. 
familiar, formulation of Theorem 1 is that a ring is a Boolean ring if and only if it is isomorphic to a ring of subsets of some set. However, we shall not discuss this aspect of the theorem since it is the subdirect sum formulation which lends itself to algebraic generalization.

3. A fundamental theorem. A problem of central interest is that of determining conditions under which a ring is isomorphic to a subdirect sum of given rings $S_{i}$. A solution of this problem is given by the next theorem which, although implicit in earlier work of Prüfer [24], Krull [14], Köthe [11], and Stone [26], was apparently first formulated explicitly in something like its present form by McCoy and Montgomery [17].

Theorem 2. $A$ ring $R$ is isomorphic to a subdirect sum of rings $S_{i}$ if and only if for each $i$ there exists a homomorphism $h_{i}$ of $R$ onto $S_{i}$ such that if $r$ is any nonzero element of $R, h_{i}(r) \neq 0$ for at least one $i$.

Proof. Let $R$ be isomorphic to a subdirect sum of rings $S_{i}$, and $h_{i}$ the natural homomorphism of $R$ onto $S_{i}$. Since the zero elements must correspond to each other under an isomorphism, not all $h_{i}(r)$ can be zero unless $r=0$. Conversely, if $h_{i}$ is a homomorphism of $R$ onto $S_{i}(i=1,2, \cdots)$ and not all $h_{i}(r)$ are zero for nonzero $r$, it is easy to verify that

$$
r \leftrightarrow\left(h_{1}(r), h_{2}(r), \cdots\right)
$$

defines an isomorphism of $R$ with a subdirect sum of the rings $S_{i}$.

From this theorem it follows easily that if $R$ is homomorphic to each of a set of rings $S_{i}{ }^{*}$ with the added condition that $R$ is isomorphic to at least one of the $S_{i}^{*}$, then $R$ has a representation as a subdirect sum of the rings $S_{i}{ }^{*}$. For this reason, if in a representation of $R$ as a subdirect sum of rings $S_{i}$ the natural homomorphism of $R$ onto $S_{i}$ is an isomorphism for at least one $i$, we say that this is a trivial representation; otherwise it is a nontrivial representation.

In view of the close relation between homomorphisms and twosided ideals, the preceding theorem may be reformulated as follows:

THEOREM 3. A ring $R$ is isomorphic to a subdirect sum of rings $S_{i}$ if and only if there exist in $R$ two-sided ideals $\mathfrak{a}_{i}$ such that $R / \mathfrak{a}_{i} \cong S_{i}$, and $\Pi \mathfrak{a}_{i}=0$.

Although Theorems 2 and 3 are fundamental in the sense that most results on subdirect sums are obtained as applications of one or the other of these theorems, it will be apparent that their principal contribution to the theory is that of stating the underlying problem 
in convenient form. In any case, the main difficulty is that of establishing the existence of the required homomorphisms or ideals.

As an application of Theorem 3, let $R$ be a commutative ring without nonzero nilpotent elements, that is, no positive integral power of a nonzero element is zero. A theorem of Krull [14] states that the set of all prime ideals in $R$ has zero intersection. Furthermore, if $\mathfrak{p}$ is a prime ideal, $R / \mathfrak{p}$ has no proper divisors of zero and is therefore an integral domain. The following result of the writer [19] then is an immediate consequence of Theorem 3:

THEOREM 4. A necessary and sufficient condition that a commutative ring $R$ be isomorphic to a subdirect sum of integral domains is that $R$ contain no nonzero nilpotent element.

4. Subdirectly irreducible rings. A ring which has no nontrivial representation as a subdirect sum of any rings is said to be subdirectly irreducible. Since a homomorphic image of a field is either a field isomorphic to the given field or a one-element ring consisting of the zero alone, it is clear that every field is subdirectly irreducible. More generally, Theorem 3 shows that $a$ ring $R$ is subdirectly irreducible if and only if the intersection of all nonzero two-sided ideals in $R$ is different from zero. Thus a subdirectly irreducible ring $R$ has a unique minimal two-sided ideal $J \neq 0$ which is contained in every nonzero two-sided ideal.

Birkhoff [2] introduced the concept of subdirect irreducibility and proved the following fundamental result:2

THeOREM 5. Any ring $R$ is isomorphic to a subdirect sum of subdirectly irreducible rings.

Proof. Let $a$ be any nonzero element of $R$, and consider the set of all two-sided ideals in $R$ which do not contain $a$. Zorn's Lemma asserts the existence of a maximal ideal $\mathfrak{m}_{a}$ in this set. If now $a$ varies over the nonzero elements of $R$ the intersection of all $\mathfrak{m}_{a}$ is zero and hence, by Theorem $3, R$ is isomorphic to a subdirect sum of the rings $R / \mathfrak{m}_{a}$. However, every nonzero two-sided ideal in $R / \mathfrak{m}_{a}$ contains $\bar{a} \neq 0$, where $\bar{a}$ is the residue class to which $a$ belongs modulo $\mathfrak{m}_{a}$, and thus $R / \mathfrak{m}_{a}$ is subdirectly irreducible.

This theorem suggests the desirability of characterizing the sub-

2 In McCoy [19] it was shown that any ring with unit element is isomorphic to a subdirect sum of irreducible rings, an irreducible ring being one which can not be expressed as the direct sum of two proper two-sided ideals. The irreducible components constructed in proving this are actually subdirectly irreducible, but the significance of this concept was first observed by Birkhoff [2]. 
directly irreducible rings. Any simple ring, that is, a ring containing no nonzero two-sided ideal except the entire ring, is clearly subdirectly irreducible. Beyond this, not much is known about the noncommutative subdirectly irreducible rings. However, in the commutative case, the subdirectly irreducible rings have been completely characterized in [21]. These results may be stated in the next two theorems, in both of which the ideal $J$ is the unique minimal ideal.

THEOREM 6. Let $R$ be a commutative ring with at least one element which is not a divisor of zero, and let $D$ be the set of all divisors of zero in $R$. Then $R$ is subdirectly irreducible if and only if it has the following properties:

(i) The set of all elements $x$ of $R$ such that $D x=0$ is a principal ideal $J=(j) \neq 0$.

(ii) $D$ is an ideal and $R / D$ is a field.

(iii) If $d_{1}$ is any element of $D$ which is not in $J$, there exists an element $d_{2}$ of $D$ not in $J$ such that $d_{1} d_{2} \in J$.

A typical example of a ring satisfying these conditions is the ring $I /\left(p^{m}\right)$ of integers modulo a power of a prime. In this case, $J=\left(p^{m-1}\right)$, $D=(p)$.

THEOREM 7. Let $R$ be a commutative ring of more than one element in which all elements are divisors of zero. Then $R$ is subdirectly irreducible if and only if it has the following properties:

(i) There exists a fixed prime $p$ such that if $a R=0$, then $p^{k} a=0$ for some positive integer $k$, depending on $a$.

(ii) The set of all elements $x$ of $R$ such that $x R=0, p x=0$, is a principal ideal $J=(j) \neq 0$.

(iii) If $b R \neq 0$, there exists an element $c$ such that $b c \in J$.

The ring of integral multiples of a prime $p$ taken modulo $p^{m}$ is an example of a ring satisfying these conditions.

In view of Theorem 5 , any commutative ring of more than one element is isomorphic to a subdirect sum of rings, each of which satisfies the conditions of Theorem 6 or those of Theorem 7.

We now observe that the representation of a ring as a subdirect sum of subdirectly irreducible rings is in no sense unique. For example, the set of all ideals $\left(p_{i}\right)$ in the ring $I$ of integers, where each $p_{i}$ is a prime of the form $4 n-1$, has zero intersection and hence $I$ is isomorphic to a subdirect sum of the fields $I /\left(p_{i}\right)$. On the other hand, it is clear that also $I$ is isomorphic to a subdirect sum of the rings $I /\left(q_{i}^{2}\right)$, where the $q_{i}$ are the primes of the form $4 n+1$. We thus have two representations of $I$ as a subdirect sum of subdirectly irreducible 
rings, all the components being fields in one representation and none being a field in the other. Actually, no component in one representation has even a subring isomorphic to a component in the other.

The condition (ii) of Theorem 7 shows that $j^{2}=0$, while condition(i) of Theorem 6 implies that $j^{2}=0$ unless $D=0$. We therefore have the following important special case due to Birkhoff [2]:

CoRollary. A subdirectly irreducible commutative ring of more than one element and without nonzero nilpotent elements is a field.

By this result, a subdirectly irreducible Boolean ring is a field, which is clearly isomorphic to $I /(2)$. Theorem 1 thus follows immediately from Theorem 5 .

Now let $R$ be a commutative ring of more than one element which is a p-ring as defined in McCoy and Montgomery [17]; that is, $p$ is a fixed prime and

$$
x^{p}=x, \quad p x=0,
$$

for every element $x$ of $R$. It will be pointed out presently that the commutativity of $R$ need not be assumed since it is a consequence of $x^{p}=x$ for every $x$. Clearly, a $p$-ring can have no nonzero nilpotent elements and the following result of McCoy and Montgomery [17] follows from the corollary and Theorem 5:

THEOREM 8. A ring $R$ is a p-ring if and only if it is isomorphic to a subdirect sum of fields $I /(p)$.

In a Boolean ring, $2 x=0$ for every element $x$ and thus a Boolean ring is just a 2-ring. Theorem 8 is therefore a generalization of Theorem 1 .

It is easy to extend Theorem 8 to the case in which

$$
x^{p^{n}}=x, \quad p x=0,
$$

for every element $x$ of $R$. This extension, as well as some related results, will be found in [18].

Another interesting application of Theorem 5 is to the regular rings of von Neumann [27, 28]. A ring $R$ is a regular ring if for each element $a$ of $R$ there exists an element $x$ of $R$ such that

$$
a x a=a .
$$

We do not require the existence of a unit element. Clearly, regular rings are generalizations of division rings since the above equation is certainly satisfied if $a$ has an inverse and we put $x=a^{-1}$.

We now consider regular rings without nonzero nilpotent elements. 
Although, in general, a homomorphic image of a ring without nonzero nilpotents may have such nilpotents, it can be shown that this does not happen in the case of regular rings. Furthermore, a subdirectly irreducible regular ring of more than one element and without nonzero nilpotent elements is a division ring. Hence we have the following result of Forsythe and McCoy [4]:

THEOREM 9. A regular ring of more than one element and without nonzero nilpotent elements is isomorphic to a subdirect sum of division rings.

As a special case of this theorem, we mention the following result of Köthe [13]:

COROLlaRy 1. Any commutative regular ring of more than one element is isomorphic to a subdirect sum of fields.

Since an algebraic algebra without nonzero nilpotent elements, as defined by Jacobson, is regular, we also have at once the result of Jacobson [9]:

Corollary 2. An algebraic algebra of more than one element and without nonzero nilpotent elements is isomorphic to a subdirect sum of division rings.

One further special case may be of interest. If the ring $R$ has the property that for each element $a$ of $R$ there exists an integer $n>1$, depending on $a$, such that

$$
a^{n}=a,
$$

then clearly $R$ is a regular ring without nonzero nilpotents and is therefore isomorphic to a subdirect sum of division rings, each of which has the same property as $R$. Jacobson [9] has proved that such a division ring is necessarily a field, that is, it is commutative. Accordingly, we have the following result (Jacobson [9], Forsythe and McCoy [4]) which is an extension of a classical theorem of Wedderburn to the effect that a division ring with a finite number of elements is necessarily commutative:

THEOREM 10. A ring $R$ with the property that for each element a there exists an integer $n>1$, depending on $a$, such that $a^{n}=a$, is necessarily commutative.

In particular, this result shows that the commutativity of a $p$-ring is a consequence of $x^{p}=x$ for every element $x$.

In addition to the references already given, other results related 
to the material of this section will be found in Brown and McCoy [3], McCoy [20], Moisil [22] and Wade [29].

5. The radical of a ring with minimum condition. The important concept of radical of a ring has usually been defined only under assumption of certain finiteness conditions, in particular, under assumption of the minimum condition. A ring $R$ satisfies the minimum condition (for right ideals) if and only if each set of right ideals in $R$ contains a minimal one, that is, an ideal containing no other one of the set. An equivalent restriction is the descending chain condition for right ideals which requires that in each sequence of right ideals $I_{i}$ such that

$$
I_{1} \supseteq I_{2} \supseteq I_{3} \supseteq \cdots,
$$

the equality must hold from some point on.

An ideal is a nil ideal if each of its elements is nilpotent. Now if $R$ is a ring which satisfies the minimum condition, the radical $N=N(R)$ of $R$ is the join (sum) of all nil right ideals in $R$. This means that $N$ consists of all elements of $R$ which are expressible in the form of a finite sum $\sum a_{i}$, where each $a_{i}$ is an element of a nil right ideal.

The following facts about the radical are to be found in the standard texts, and we state them briefly in preparation for some generalizations to be discussed presently. The radical of $R$ is a two-sided ideal in $R$ which contains every nil ideal (right, left, or two-sided). If $N(R)=0, R$ is said to be semi-simple, and the structure of semi-simple rings is given by the following classical results of Wedderburn and Artin. A ring $R$ satisfying the minimum condition is semi-simple if and only if it is the direct sum of (a finite number of) simple rings. Furthermore, each of these simple rings is isomorphic to a complete matrix ring over a division ring since a simple ring with minimum condition is necessarily of this type. It is also known that the residue class ring $R / N$ is always semi-simple and thus has the structure just described.

If we relax the requirement that $R$ must satisfy the minimum condition, the results as stated are no longer valid. Various attempts have been made to define a useful radical for an arbitrary ring, most of them quite naturally in terms of nilpotence. ${ }^{3}$ However, it now appears that other concepts are probably more fundamental in the definition of radical. We shall presently define two different radicals of a general ring, both of which coincide with the radical as defined above in case the minimum condition is assumed, and both of which.lead to

\footnotetext{
${ }^{3}$ See Jacobson $[8]$ for references.
} 
subdirect sum decompositions which generalize the direct sum decomposition mentioned above.

Before proceeding, we may remark that a regular ring which satisfies the minimum condition is necessarily semi-simple (von Neumann [28]). Thus the regular rings, although not involving the concept of radical, do furnish a generalization of the notion of semi-simple ring.

6. The Jacobson radical. We now turn to a brief discussion of the radical of a general ring as defined by Jacobson [8], based partly on previous work of Perlis [23] and Baer [1].

If $a$ is an element of the arbitrary ring $R$, the set of all elements of the form $x+a x, x \in R$, is a right ideal $\{x+a x\}$ in $R$. If $a \in\{x+a x\}, a$ is said to be quasi-regular, and an ideal is quasi-regular if and only if each of its elements is quasi-regular. The Jacobson radical $N_{1}=N_{1}(R)$ is defined to be the join of all quasi-regular right ideals in $R$.

If $z^{n}=0$, it is easy to verify that $z=x+z x$, where

$$
x=-\sum_{i=1}^{n-1}(-z)^{i}
$$

and thus every nilpotent element is quasi-regular. Hence every nil ideal is a quasi-regular ideal and $N_{1}$ contains every nil right ideal of $R$. However, $N_{1}$ may well contain elements which are not nilpotent -in contrast to the radical $N$ as defined in the preceding section for rings with minimum condition. For example, if $S$ is the ring of rational numbers of the form $r / s$, where $s$ is odd, it is easy to verify that $N_{1}(S)=(2) \neq 0$, and $S$ has no nonzero nilpotent elements.

Jacobson [8] has proved the following facts. The radical $N_{1}=N_{1}(R)$ is a two-sided ideal in $R$, and $R / N_{1}$ is semi-simple in the sense that $N_{1}\left(R / N_{1}(R)\right)=0$. If $R$ satisfies the minimum condition, then $N_{1}(R)$ $=N(R)$, and thus the Jacobson radical is actually a generalization of the ordinary radical $N$. If $R_{n}$ denotes the complete matrix ring of order $n$ with elements in $R$, then $N_{1}\left(R_{n}\right)=\left(N_{1}(R)\right)_{n}$. Furthermore, if $R$ is a regular ring, then $N_{1}(R)=0$, that is, a regular ring is semisimple.

Before stating the result which shows the fundamental connection of the radical with the notion of subdirect sum, we need some preliminary definitions of Jacobson [7]. Let $\mathfrak{M}$ be a vector space, of any finite or infinite dimension, over a division ring $D$, and let $S$ be a ring of linear transformations of $\mathfrak{M}$. If $a \in S$, the effect of applying the linear transformation $a$ to the vector $x$ of $\mathfrak{M}$ may be denoted by $x a$. Suppose now that in $\mathfrak{M}$ there exist at least $k$ vectors which are linearly independent relative to $D$. The ring $S$ is said to be $k$-fold 
transitive if for every set $x_{1}, x_{2}, \cdots, x_{k}$ of $k$ linearly independent vectors and arbitrary vectors $y_{1}, y_{2}, \cdots, y_{k}$, there exists an element $b$ of $S$ such that $x_{i} b=y_{i}(i=1,2, \cdots, k)$. The ring $S$ is dense in $\mathfrak{M}$ over $D$ if it is $k$-fold transitive for all $k=1,2, \cdots$ (up to the dimension of $\mathfrak{M}$ if $\mathfrak{M}$ has finite dimension). The abbreviated statement that $S$ is a dense ring is to be interpreted as meaning that $S$ is a dense ring of linear transformations of some vector space over a division ring. [8]:

We can now state the following fundamental theorem of Jacobson

THEOREM 11. A necessary and sufficient condition that a ring $R$ be isomorphic to a subdirect sum of dense rings is that $N_{1}(R)=0 .{ }^{4}$

Jacobson [7] has pointed out that a dense ring $S$ satisfies the minimum condition if and only if its vector space $\mathfrak{M}$ has finite dimension. Furthermore, a dense ring of linear transformations of an $n$-dimensional vector space is the ring of all linear transformations of the space and hence is isomorphic to the ring of all matrices of order $n$ with elements in a division ring which is anti-isomorphic to the underlying division ring. This shows that the dense rings generalize the complete matrix rings over division rings. Theorem 11 is thus an appropriate analogue of one of the Wedderburn-Artin theorems for rings with minimum condition.

It is easy to see that a commutative dense ring is necessarily a field and Theorem 8, for example, may be obtained as a corollary to Theorem 11 since a $p$-ring is necessarily semi-simple.

Before proceeding to still another definition of radical, we may make a few additional remarks. In the first place, we point out that a dense ring need not be simple since the (dense) ring of all linear transformations of an infinite dimensional vector space contains as a proper two-sided ideal the set of all finite valued transformations $a$, that is, transformations $a$ such that $\mathfrak{M} a$ has finite dimension. However, an important result of Jacobson [7] shows that a simple ring with a maximal right ideal - in particular, a simple ring with unit elementis isomorphic to a dense ring. In this connection, we may also observe that a dense ring need not have a unit element since the ring of all finite valued linear transformations of an infinite dimensional vector space is itself a dense ring without unit element.

In a recent paper, Goldman [5] has discussed the radical of a

${ }^{4}$ Jacobson has formulated this result in terms of primitive rings rather than dense rings, but has shown in [8] that the concepts are equivalent. If $I$ is a right ideal in $R$, we may denote by $I: R$ the set of all elements $a$ of $R$ such that $R a \subseteq I$. The ring $R$ is said to be primitive if there exists in $R$ a maximal right ideal $I$ such that $I: R=0$. 
general ring, using a definition which he attributes to Chevalley. According to this definition, the radical of $R$ is the intersection of the annihilators of all simple $R$-modules. It can be shown, however, that this coincides with $N_{1}(R)$ and hence we have an interesting alternative definition of $N_{1}(R)$.

7. Another definition of radical. In preparation for the definition to be stated presently, let us give alternate characterizations of the previously defined radicals as follows.

If $R$ satisfies the minimum condition, the radical $N(R)$ consists of the elements $a$ of $R$ such that every element of the principal twosided ideal $(a)$ is nilpotent.

For arbitrary $R$, the Jacobson radical $N_{1}(R)$ consists of the elements $a$ of $R$ such that every element of the principal two-sided ideal $(a)$ is quasi-regular. That is, for every element $b$ of $(a)$, we have $b \in\{x+b x\}$.

We now denote by $G(b)$ the two-sided ideal in $R$ generated by the elements of the right ideal $\{x+b x\}$, that is,

$$
G(b)=\left\{t+b t+\sum y_{i} z_{i}+\sum y_{i} b z_{i}\right\},
$$

where $t, y_{i}, z_{i}$ vary over $R$, the sums naturally being finite. We define the radical $N_{2}(R)$ to consist of the elements $a$ of $R$ such that $b \in G(b)$ for every element $b$ of the principal two-sided ideal $(a)$. This definition and the results to be presently stated concerning this radical are to be found in Brown and McCoy [3].

It is clear that always $N_{1}(R) \subseteq N_{2}(R)$, and if $R$ happens to be commutative, $N_{1}(R)=N_{2}(R)$. Furthermore, it can be shown that the equality also holds if $R$ satisfies the minimum condition. However, we shall presently give an example which will show that in general $N_{1}(R)$ and $N_{2}(R)$ do not coincide; hence they are different generalizations of the radical $N(R)$.

It can be shown that $N_{2}(R)$ is a two-sided ideal in $R$, and that $R / N_{2}(R)$ is semi-simple in the sense that $N_{2}\left(R / N_{2}(R)\right)=0$. Also, if $R_{n}$ is the complete matrix ring of order $n$ over $R$, then $N_{2}\left(R_{n}\right)$ $=\left(N_{2}(R)\right)_{n}$. Actually, $N(R), N_{1}(R)$ and $N_{2}(R)$ have these properties in common.

If $R$ has more than one element and a unit element $1, G(-1)=0$; and hence $N_{2}(R) \neq R$. If $S$ is a simple ring with unit element it therefore follows that $N_{2}(S)=0$. The following result shows that simple rings with unit element are of fundamental interest in a study of this radical :

TheOREM 12. A necessary and sufficient condition that a ring $R$ be 
isomorphic to a subdirect sum of simple rings with unit element is that $N_{2}(R)=0$.

The main step in the proof of this theorem is that of showing that $N_{2}(R)$ may be characterized as the intersection of all two-sided ideals $\mathfrak{a}$ in $R$ such that $R / \mathfrak{a}$ is a simple ring with unit element. ${ }^{5}$ One part of the theorem then follows at once from Theorem 3, and the other part follows from the observation that if $R$ is homomorphic to $S$, then $N_{2}(R)$ goes into $N_{2}(S)$ under the given homomorphism.

It can be shown that a subdirect sum of a finite number of simple rings is isomorphic to the direct sum of some or all of these rings. From this, and the preceding theorem, it is easy to obtain the following corollary.

COROLlary. Let $R$ be a ring in which the descending chain condition is satisfied for two-sided ideals. A necessary and sufficient condition that $R$ be isomorphic to a direct sum of a finite number of simple rings with unit element is that $N_{2}(R)=0$.

An example may clarify the relation between $N_{1}(R)$ and $N_{2}(R)$. Let $\mathbb{R}$ be the ring of all linear transformations of a vector space $\mathfrak{M}$ with a denumerable basis over a division ring, and $\mathfrak{F}$ the set of all finitevalued transformations of $\mathbb{R}$. Jacobson [7] has shown that $\mathfrak{F}$ is a two-sided ideal of $\mathbb{R}$ which is contained in every nonzero two-sided ideal of $\mathfrak{R}$, and hence $\mathfrak{R}$ is subdirectly irreducible. Furthermore, Johnson and Kiokemeister [10] have pointed out that $\mathfrak{F}$ is the only proper two-sided ideal in $\mathfrak{R}$. Now $N_{2}(\mathfrak{R}) \neq \mathfrak{R}$ since $\mathfrak{R}$ has a unit element, and hence $N_{2}(\mathfrak{R})=\mathfrak{F}$ or $N_{2}(\mathfrak{R})=0$. If $N_{2}(\mathfrak{R})$ were zero, the preceding theorem would show that $\Omega$ is isomorphic to a subdirect sum of simple rings with unit element. However, since $\mathfrak{R}$ is subdirectly irreducible, this would imply that $\mathfrak{R}$ is itself a simple ring with unit element, which is obviously false. Thus $N_{2}(R)=\mathfrak{F}$, and it is also easy to verify that $N_{2}(\mathfrak{F})=\mathfrak{F}$. Since $\mathfrak{l}$ and $\mathfrak{F}$ are dense rings, we have $N_{1}(\mathfrak{R})=N_{1}(\mathfrak{F})$ $=0$, and therefore $N_{1}$ is properly contained in $N_{2}$ in the case of both the rings $\mathfrak{R}$ and $\mathfrak{F}$.

Incidentally, Johnson and Kiokemeister [10] have shown that the ring of all linear transformations of any vector space is a regular ring, and since $N_{2}(\Omega) \neq 0$ we see that a regular ring need not be semi-

${ }^{5}$ Cf. Segal [25] who essentially takes this as the definition of the radical of a group algebra. We may point out that a one-element ring is here considered as a simple ring with unit element, so that $N_{2}(R)=R$ in case there exists no maximal two-sided ideal $\mathfrak{a}$ such that $R / \mathfrak{a}$ has a unit element. If we complete Segal's definition by agreeing that his radical is to be the entire ring $R$ in the case just described, then Segal's radical and $N_{2}(R)$ actually coincide for every ring $R$. 
simple with reference to the last defined radical. We may also remark that $\mathfrak{R}$ is a regular ring which can not be isomorphic to a subdirect sum of simple regular rings. ${ }^{6}$

As another example, it may be of interest to state that in the ring $A$ of all bounded operators in Hilbert space, $N_{1}(A)=0$ while $N_{2}(A)$ is the ideal of all completely continuous operators.

In the definition of $N_{2}(R)$ one may just as well use in place of $G(b)$ the ideal

$$
G^{\prime}(b)=\left\{t+t b+\sum y_{i} z_{i}+\sum y_{i} b z_{i}\right\}
$$

or the ideal

$$
G^{\prime \prime}(b)=\left\{s+b s+t+t b+\sum y_{i} z_{i}+\sum y_{i} b z_{i}\right\} .
$$

Each of these lead to precisely the same radical $N_{2}(R)$. In case $R$ happens to have a unit element 1 , clearly

$$
G(b)=G^{\prime}(b)=G^{\prime \prime}(b)=\left\{\sum y_{i}(1+b) z_{i}\right\} .
$$

8. The $F$-radical of a ring. Most of the results of the preceding section have been generalized as follows by Brown and McCoy [3]. Let $b \rightarrow F(b)$ be a mapping $F$ of $R$ into the set of two-sided ideals in $R$ which is defined for all rings and has the property that if $b \rightarrow \bar{b}$ is a homomorphism of $R$ onto a ring $S$, then

$$
F(\bar{b})=\overline{F(b)} \text {. }
$$

The mapping $b \rightarrow G(b)$, where $G(b)$ is the two-sided ideal defined above, clearly has this property. If in the definition of $N_{2}(R)$ we replace $G(b)$ by $F(b)$ we get what we shall call the $F$-radical $N_{F}$ of $R$.

It can be shown that $N_{F}$ is a two-sided ideal in $R$ which may be characterized as the intersection of all two-sided ideals $\mathfrak{a}$ such that $R / \mathfrak{a}$ is subdirectly irreducible and has zero $F$-radical. Also, $R / N_{F}$ has zero $F$-radical. Concerning subdirect sums we have the following theorem.

ThEOREM 13. A ring has zero F-radical if and only if it is isomorphic to a subdirect sum of subdirectly irreducible rings of zero F-radical.

These results show that the $F$-radical has a number of "radicallike" properties. However, in general, it need not reduce to the radical $N$ in case the minimum condition is satisfied in $R$, and hence is not an actual generalization of that radical.

Let us now consider a few illustrations. If $F(b)$ is defined by

${ }^{8}$ This apparently contradicts a statement of Köthe [13, p. 141]. 
$F(b)=0$ for all elements $b$ of a ring, then every ring has zero $F$-radical and Theorem 13 reduces to Birkhoff's Theorem 5. If we define $F(b)=G(b)$, it is not difficult to show that a subdirectly irreducible ring of zero $F$-radical is a simple ring with unit element, and Theorem 13 yields Theorem 12 .

As another illustration, suppose that

$$
F_{1}(b)=G\left(b^{2}\right)=\left\{t+b^{2} t+\sum y_{i} z_{i}+\sum y_{i} b^{2} z_{i}\right\} .
$$

In this case it turns out that a subdirectly irreducible ring of zero $F_{1}$-radical is a simple ring with unit element 1 and an element whose square is -1 . Hence Theorem 13 gives us the following rather curious result:

Theorem 14. A ring has zero $F_{1}$-radical if and only if it is isomorphic to a subdirect sum of simple rings, each with a unit element 1 and an element whose square is -1 .

A corresponding result will be obtained if in the definition of $F_{1}(b)$ we replace $b^{2}$ by any fixed polynomial in $b$ with integral coefficients and zero constant term. For other illustrations of the general theory, see Brown and McCoy [3].

9. Special subdirect sums. The earliest work on subdirect sums was not concerned with subdirect sums as we have so far discussed them but rather with subdirect sums in which additional restrictions are imposed. In the remainder of this address we shall give a brief exposition of some results concerning these restricted subdirect sums. The material of the present section is primarily due to Köthe [11].

Let $S$ be the direct sum of rings $S_{i}$, the elements of which we may denote as usual by

$$
\left(s_{1}, s_{2}, \cdots\right) \quad\left(s_{i} \in S_{i}, i=1,2, \cdots\right) .
$$

Now let $S_{1}^{\prime}$ be the subring of $S$ consisting of all elements of $S$ of the form

$$
\left(s_{1}, 0,0, \cdots\right) \quad\left(s_{1} \in S_{1}\right),
$$

and similarly $S_{i}^{\prime}$ is the subring of $S$ consisting of all elements with an element of $S_{i}$ in the $i$ th "place" and zeros elsewhere. We shall say that a subdirect sum $T$ of the rings $S_{i}$ is a special subdirect sum ${ }^{7}$ if $T$ contains all $S_{i}^{\prime}(i=1,2, \cdots)$. In this case, the rings $S_{i}^{\prime}$ are actually two-sided ideals in $T$. Furthermore, the set of all elements of $T$ with

${ }^{7}$ Köthe calls this a "transcendental direct sum." 
0 in the $i$ th "place" is also a two-sided ideal $T_{i}^{\prime}$ and, for each $i$,

$$
T=S_{i}^{\prime}+T_{i}^{\prime},
$$

that is, $T$ is the direct sum of its ideals $S_{i}^{\prime}$ and $T_{i}^{\prime}$.

Before stating the conditions under which a ring $R$ is isomorphic to a special subdirect sum of given two-sided ideals in $R$, we need a result of a very special nature. If $x$ is an element of $R$ such that $x R=R x=0$, we may say that $x$ is an annihilator of $R$.

LEMMA 1. Let $R$ be a ring with no nonzero annihilator. If $\mathfrak{a}_{1}, \mathfrak{a}_{2}$, $\mathfrak{b}_{1}, \mathfrak{b}_{2}$ are two-sided ideals in $R$ such that $\mathfrak{a}_{1} \cap \mathfrak{a}_{2}=0$, and $R=\mathfrak{a}_{1}+\mathfrak{b}_{1}$ $=\mathfrak{a}_{2}+\mathfrak{b}_{2}$, then $\mathfrak{a}_{1} \subseteq \mathfrak{b}_{2}$.

Proof. Let $a_{1}$ be any element of $\mathfrak{a}_{1}$. Since $R=\mathfrak{a}_{2} \dot{+} \mathfrak{b}_{2}$, we may write

$$
a_{1}=a_{2}+b_{2}
$$

where $a_{2} \in \mathfrak{a}_{2}, b_{2} \in \mathfrak{b}_{2}$. If $x$ is any element of $\mathfrak{a}_{2}$, then $x a_{1} \in \mathfrak{a}_{1} \cap \mathfrak{a}_{2}=0$, and similarly $x b_{2}=0$ since $\mathfrak{a}_{2} \cap \mathfrak{b}_{2}=0$. It follows that $x a_{2}=0$, that is, $\mathfrak{a}_{2} a_{2}=0$. From $R=\mathfrak{a}_{2}+\mathfrak{b}_{2}$, it then follows that $R a_{2}=0$. In like manner, it can be shown that $a_{2} R=0$, and hence we must have $a_{2}=0$ and $a_{1}=b_{2} \in \mathfrak{b}_{2}$.

By a suitable change of notation, the lemma also shows that under the given hypothesis on $R, R=\mathfrak{a}_{1}+\mathfrak{b}_{1}=\mathfrak{a}_{1}+\mathfrak{b}_{2}$ implies that $\mathfrak{b}_{1}=\mathfrak{b}_{2}$.

The following theorem is due to Köthe [11] except for the restriction on $R$ which seems to be necessary at one point in the proof.

THEOREM 15. Let $R$ be a ring with no nonzero annihilator, and $\mathfrak{a}_{i}(i=1,2, \cdots)$ a set of two-sided ideals in $R$. Necessary and sufficient conditions that $R$ be isomorphic to a special subdirect sum of the $\mathfrak{a}_{i}$ are:

(i) $\mathfrak{a}_{i} \cap \mathfrak{a}_{j}=0$ for $i \neq j$.

(ii) For each $i, \mathfrak{a}_{i}$ is a direct component of $R$, that is, there exists a (unique) two-sided ideal $\mathfrak{b}_{i}$ in $R$ such that $R=\mathfrak{a}_{i}+\mathfrak{b}_{i}$.

(iii) $\prod \mathfrak{b}_{i}=0$.

The necessity of these conditions follows readily from the observations made above-without any restriction on $R$. Clearly, these conditions are satisfied for the special subdirect sum $T$ by identifying $S_{i}^{\prime}$ with $\mathfrak{a}_{i}, T_{i}^{\prime}$ with $\mathfrak{b}_{i}$. The same conditions obviously hold for the corresponding ideals of any ring $R$ which is isomorphic to $T$.

To prove the converse, suppose that ideals $\mathfrak{a}_{i}$ in $R$ satisfy the three conditions of the theorem. If $a$ is any element of $R$, then by (ii) we can express $a$ uniquely in the form

$$
a=a_{i}+b_{i} \quad\left(a_{i} \in \mathfrak{a}_{i}, b_{i} \in \mathfrak{b}_{i}\right) .
$$


Let us denote the homomorphism $a \rightarrow a_{i}$ of $R$ onto $\mathfrak{a}_{i}$ by $h_{i}$, and write $h_{i}(a)=a_{i}$. If $a$ is different from zero, $a$ is not in some $\mathfrak{b}_{i}$ by (iii) and hence, for some $i, a_{i}=h_{i}(a) \neq 0$. As in the proof of Theorem 2 it follows that

$$
a \leftrightarrow\left(h_{1}(a), h_{2}(a), \cdots\right)
$$

is an isomorphism of $R$ with a subdirect sum of the $\mathfrak{a}_{i}$. Moreover, Lemma 1 shows that $\mathfrak{a}_{i} \subseteq \mathfrak{b}_{j}$ for $j \neq i$, and hence if $c \in \mathfrak{a}_{i}$ then $h_{i}(c)=c$, $h_{j}(c)=0$ for $j \neq i$. Thus we actually have a special subdirect sum, and the proof is completed.

It may be of interest to point out that the theorem is not true without some restriction on $R$. As an example, let $R$ be the ring with the four distinct elements $0, a, b, a+b$; it being given that $x y=0$ for all elements $x, y$ of $R$ and that $2 x=0$ for every element $x$. Then the ideals $\mathfrak{a}_{1}=\{0, a\}, \mathfrak{a}_{2}=\{0, b\}, \mathfrak{a}_{3}=\{0, a+b\}$ satisfy the three conditions of the theorem with $\mathfrak{b}_{1}=\{0, b\}, \mathfrak{b}_{2}=\{0, a+b\}$, $\mathfrak{b}_{3}=\{0, a\}$. In this case, however, the $\mathfrak{b}_{i}$ are not unique and furthermore $R$ can not be isomorphic to a special subdirect sum of the ideals $\mathfrak{a}_{1}, \mathfrak{a}_{2}, \mathfrak{a}_{3}$ since a special subdirect sum of a finite number of rings is actually the direct sum, and the direct sum of these three rings has eight distinct elements.

We shall presently apply Theorem 15 to prove another result, but first we need a few preliminary remarks. If, considered as a ring, the two-sided ideal $a$ in $R$ has a unit element $e$, we may say that $e$ is the unit element of the ideal $a$.

Lemma 2. If the two-sided ideal $\mathfrak{a}$ has a unit element, $\mathfrak{a}$ is a direct component of $R$.

Proof. If $e$ is the unit element of $a$, then $e y=e y e=y e$ for every $y$ in $R$, and hence $e$ commutes with all elements of $R$. If $\mathfrak{b}=\{x-e x\}$, it is easy to show that $R=\mathfrak{a}+\mathfrak{b}$. Furthermore, $\mathfrak{b}$ may be characterized as the set of all elements $b$ of $R$ such that $e b=b e=0$.

A nonzero two-sided ideal in $R$ which does not properly contain a nonzero two-sided ideal of $R$ may be called a simple ideal. Thus a simple ideal is just a minimal nonzero two-sided ideal. The next result is an immediate consequence of Lemma 2 :

Lemma 3. A simple ideal of $R$ which has a unit element is a simple ring.

We may now prove the following theorem.

THEOREM 16. A necessary and sufficient condition that a ring $R$ be 
isomorphic to a special subdirect sum of simple rings with unit element is that every nonzero two-sided ideal in $R$ contain a simple ideal with unit element.

We first establish the sufficiency of this condition. If $a$ is an annihilator of $R$, the principal two-sided ideal (a) can contain no simple ideal with unit element; hence $R$ has no nonzero annihilator. Let $\mathfrak{a}_{i}(i=1,2, \cdots)$ be the set of all simple ideals in $R$ with unit element. By Lemma $2, R=\mathfrak{a}_{i}+\mathfrak{b}_{i}$, where $\mathfrak{b}_{i}$ consists of all elements $x$ of $R$ such that $e_{i} x=x e_{i}=0, e_{i}$ being the unit element of $a_{i}$. Furthermore, $\mathfrak{a}_{i} \cap \mathfrak{a}_{j}=0$ for $i \neq j$, since $\mathfrak{a}_{i} \cap \mathfrak{a}_{j}$ is an ideal in the simple ideal $\mathfrak{a}_{i}$ (or $\mathfrak{a}_{j}$ ). Thus the conditions (i) and (ii) of Theorem 15 are satisfied, and there remains to verify that $\prod \mathfrak{b}_{i}=0$. If $\prod \mathfrak{b}_{i} \neq 0$, it is a nonzero two-sided ideal of $R$ and hence, by hypothesis, it contains some $\mathfrak{a}_{i}$, say $\mathfrak{a}_{1}$. Hence $\prod \mathfrak{b}_{i}$ contains the unit element $e_{1}$ of $\mathfrak{a}_{1}$. But $\prod \mathfrak{b}_{i} \subseteq \mathfrak{b}_{1}$ and thus $e_{1} \prod \mathfrak{b}_{i}=0$, from which it follows that $e_{1}^{2}=e_{1}=0$, and $\mathfrak{a}_{1}=0$. This is impossible since, by definition, a simple ideal is different from zero. Hence $\Pi \mathfrak{b}_{i}=0$, and Theorem 15 shows that $R$ is isomorphic to a special subdirect sum of the simple rings $\mathfrak{a}_{i}$ with unit element.

Conversely, suppose that $R$ is isomorphic to a special subdirect sum $T$ of simple rings $S_{i}$ with unit element. We shall show that every nonzero ideal in $T$ contains a simple ideal with unit element, and the same will therefore be true for $R$. Let $\mathfrak{b}$ be a nonzero two-sided ideal in $T$, and $b$ a nonzero element of $\mathfrak{b}$, say

$$
b=\left(b_{1}, b_{2}, b_{3}, \cdots\right) \text {, }
$$

where $b_{i} \in S_{i}(i=1,2, \cdots)$. Since $b \neq 0$, some $b_{i} \neq 0$ and suppose, for convenience of notation, that $b_{1} \neq 0$. Then $\mathfrak{b}$ contains

$$
\left(b_{1}, b_{2}, b_{3}, \cdots\right)(1,0,0, \cdots)=\left(b_{1}, 0,0, \cdots\right),
$$

and therefore contains the two-sided ideal generated by $\left(b_{1}, 0,0, \cdots\right)$. However, since $S_{1}$ is a simple ring this is just the set of all elements $(x, 0,0, \cdots), x \in S_{1}$. This is therefore a simple ring with unit element, and the proof is completed.

We conclude our remarks on special subdirect sums with a result of Köthe [11]. A ring with minimum condition which contains no nonzero nil ideal is sometimes said to be completely reducible. More generally, a two-sided ideal $\mathfrak{a}$ in the arbitrary ring $R$ may be said to be completely reducible if it contains no nonzero nil ideal of $R$ and each set of right ideals of $R$ which are contained in $\mathfrak{a}$ has a minimal one. It can be shown that a completely reducible two-sided ideal $\mathfrak{a}$ of $R$ has a unit element and is therefore a direct component of $R$. Thus $\mathfrak{a}$ is 
also as a ring completely reducible and hence, by the results stated in $\$ 5$, is a direct sum of simple rings each of which is isomorphic to a complete matrix ring over a division ring.

THEOREM 17. A necessary and sufficient condition that a ring $R$ be isomorphic to a special subdirect sum of simple rings, each of which is a complete matrix ring over a division ring, is that every two-sided ideal in $R$ contain a completely reducible two-sided ideal of $R$.

The proof follows by arguments similar to those used in the proof of Theorem 16 except that the set of all simple ideals satisfying the minimum condition takes the place of the set of all simple ideals with unit element which was used in the proof of that theorem.

10. Discrete direct sums. As usual, let $S$ be the direct sum of rings $S_{i}$, with elements

$$
s=\left(s_{1}, s_{2}, \cdots\right) \quad\left(s_{i} \in S_{i}\right) .
$$

The subring $T$ of $S$ which consists of all elements $s$ such that $s_{i}$ is different from zero for only a finite number of values of $i$ may be called the discrete direct sum of the rings $S_{i}$. It will be observed that a discrete direct sum is a restricted case of a special subdirect sum, and that a discrete direct sum of a finite number of rings is actually the direct sum. Furthermore, if $R$ is isomorphic to a special subdirect sum of its ideals $\mathfrak{a}_{i}(i=1,2, \cdots), R$ will clearly be isomorphic to the discrete direct sum of the $\mathfrak{a}_{i}$ if and only if $R$ is the join of the ideals $\mathfrak{a}_{i}$. Therefore, a ring $R$ without nonzero annihilator is isomorphic to a discrete direct sum of ideals $\mathfrak{a}_{i}$ if and only if $R$ is the join of these ideals and the three conditions of Theorem 15 are satisfied. As an application of these observations, we can readily prove the following theorem.

THEOREM 18. A necessary and sufficient condition that a ring $R$ be isomorphic to a discrete direct sum of simple rings with unit element is that $R$ be the join of its simple two-sided ideals with unit element.

The necessity of the condition is almost obvious and we therefore consider only the sufficiency. Let $R$ be the join of its simple two-sided ideals $\mathfrak{a}_{i}$ with unit element $e_{i}$. Conditions (i) and (ii) of Theorem 15 are satisfied as shown in the proof of Theorem 16, and $\mathfrak{b}_{i}$ is the set of all elements $b$ of $R$ such that $e_{i} b=b e_{i}=0$. Now each element $a$ of $R$ is expressible as a finite sum

$$
a=a_{i_{1}}+a_{i_{2}}+\cdots+a_{i_{k}} \quad\left(a_{i_{s}} \in \mathfrak{a}_{i_{s}}, s=1,2, \cdots, k\right) .
$$


Since $\mathfrak{a}_{i} e_{j} \subseteq \mathfrak{a}_{i} \cap \mathfrak{a}_{j}$, we see that $\mathfrak{a}_{i} e_{j}=0$ for $i \neq j$. Thus

$$
a\left(e_{i_{1}}+e_{i_{2}}+\cdots+e_{i_{k}}\right)=a,
$$

and $R$ can have no nonzero annihilator. Suppose now that $a \in \prod \mathfrak{b}_{i}$. Since $a e_{i_{1}}=a_{i_{1}}$, it follows that $a_{i_{1}} \in \prod \mathfrak{b}_{i} \subseteq \mathfrak{b}_{i_{1}}$. But $\mathfrak{a}_{i_{1}} \cap \mathfrak{b}_{i_{1}}=0$, and hence $a_{i_{1}}=0$. Similarly, each $a_{i_{\varepsilon}}=0$, and therefore $a=0$, that is, $\prod \mathfrak{b}_{i}=0$. Thus all the conditions of Theorem 15 are satisfied, and this completes the proof.

If $R$ happens to be a Boolean ring, a simple ideal is a field of two elements, and $R$ is the join of its simple ideals with unit element if and only if $R$ has an atomic basis as defined by Stone. The case of Theorem 18 in which $R$ is restricted to be a Boolean ring was proved by Stone [26].

For another result which is related to the material of this section, see Jacobson [8, p. 319].

11. Ring imbedding. If a ring $R$ is isomorphic to a subring of a direct sum $S$ of rings $S_{i}$, we may identify $R$ with this isomorphic subring and consider that $S$ contains $R$. Furthermore, there may be important subrings of $S$ which also contain $R$.

In the first place, we may point out that one may sometimes get an interesting result by simply considering $R$ as imbedded in the direct sum $S$ itself. For example, since a ring which is dense in a vector space $\mathfrak{M}$ over a division ring is a subring of the regular ring of all linear transformations of $\mathfrak{M}$, and a direct sum of regular rings is a regular ring, Theorem 11 yields the following result:

THEOREM 19. If $N_{1}(R)=0, R$ is a subring of a regular ring.

Since the radical as defined by Goldman [5] coincides with $N_{1}(R)$, the results of Goldman [6] furnish necessary and sufficient conditions that a ring be a subring of a regular ring. It may be of interest to remark that from these conditions it is easy to show that there exist commutative rings which can be imbedded in regular rings but not in commutative regular rings.

We now describe an imbedding procedure of a different nature. Let $R$ be a ring which is isomorphic to a subdirect sum of rings $S_{i}$, and let $h_{i}$ be the natural homomorphism of $R$ onto $S_{i}$. We consider the set $T_{1}$ of all elements

$$
t=\left(t_{1}, t_{2}, \cdots\right)
$$

of the direct sum of the rings $S_{i}$ with the property that if $i_{1}, i_{2}, \cdots, i_{n}$ 
is $a n y$ finite set of distinct indices, there exists an element $r$ of $R$ (depending on the set of indices) such that

$$
h_{i_{k}}(r)=t_{i_{k}} \quad(k=1,2, \cdots, n) .
$$

That is, at any finite number of "places," $t$ coincides with some element of $R$. It is easily verified that $T_{1}$ is a subring of $S$ which contains $R$.

In principle, the method by which $R$ has been imbedded in $T_{1}$ is due to Prüfer [24]. Although his point of view was somewhat different, he was apparently the first to use any kind of subdirect sum representation. Prüfer was concerned with the case in which $R$ is the ring of algebraic integers of a finite algebraic number field and, in our terminology, pointed out that $R$ is isomorphic to a subdirect sum of rings $R /(a)$, where $a$ varies over the nonzero elements of $R$. He then showed that the ring $T_{1}$, constructed as described above, is a ring containing $R$ and that in $T_{1}$ each element of $R$ is uniquely expressible as a product of prime factors. In other words, the elements of $T_{1}$ are "ideal numbers" whose adjunction to $R$ restores unique factorization of elements of $R$.

By an imbedding process somewhat similar to that just described, it is possible to imbed the ring of rational integers in the ring of $p$-adic integers. This is carried out in detail in Krull [16] where reference is made to Prüfer [24]. More recently, the same general idea has been used in various connections-in particular, in certain parts of the class field theory.

\section{BIBLIOGRAPHY}

1. R. Baer, Radical ideals, Amer. J. Math. vol. 65 (1943) pp. 537-568.

2. G. Birkhoff, Subdirect unions in universal algebra, Bull. Amer. Math. Soc. vol. 50 (1944) pp. 764-768.

3. B. Brown and N. H. McCoy, Radicals and subdirect sums, Amer. J. Math. vol. 69 (1947) pp. 46-58.

4. A. Forsythe and N. H. McCoy, On the commutativity of certain rings, Bull. Amer. Math. Soc. vol. 52 (1946) pp. 523-526.

5. $\mathrm{O}$. Goldman, $A$ characterization of semi-simple rings with the descending chain condition, Bull. Amer. Math. Soc. vol. 52 (1946) pp. 1021-1027.

6. - Semi-simple extensions of rings, Bull. Amer. Math. Soc. vol. 52 (1946) pp. 1028-1032.

7. N. Jacobson, Structure theory of simple rings without finiteness assumptions, Trans. Amer. Math. Soc. vol. 57 (1945) pp. 228-245.

8. - The radical and semi-simplicity for arbitrary rings, Amer. J. Math. vol. 67 (1945) pp. 300-320.

9. - Structure theory for algebraic algebras of bounded degree, Ann. of Math. vol. 46 (1945) pp. 695-707.

10. R. E. Johnson and F. L. Kiokemeister, The endomorphisms of the total operator domain of an infinite module, to appear in Trans. Amer. Math. Soc. 
11. G. Köthe, Abstrakte Theorie nichkommutativer Ringe mit einer Anwendung auf die Darstellungstheorie kontinuierlicher Gruppen, Math. Ann. vol. 103 (1930) pp. 545572.

12. - Ein Beitrag zur Theorie der kommutativen Ringe ohne Endlichkeitsbedingung, Nachr. Ges. Wiss. Göttingen (1930) pp. 195-207.

13. - Die Theorie der Verbände, Jber. Deutschen Math. Verein. vol. 47 (1937) pp. 125-144.

14. W. Krull, Idealtheorie in Ringen ohne Endlichkeitsbedingung, Math. Ann. vol. 101 (1929) pp. 729-744.

15. - Idealtheorie, Berlin, 1935.

16. —, Ein Satz ïber primäre Integritätsbereiche, Math. Ann. vol. 103 (1930) pp. $450-465$.

17. N. H. McCoy and D. Montgomery, A representation of generalized Boolean rings, Duke Math. J. vol. 3 (1937) pp. 455-459. 382.

18. N. H. McCoy, Subrings of direct sums, Amer. J. Math. vol. 60 (1938) pp. 374-

19. - Subrings of infinite direct sums, Duke Math. J. vol. 4 (1938) pp. 486494.

20. - Generalized regular rings, Bull. Amer. Math. Soc. vol. 45 (1939) pp. $175-178$.

21. - Subdirectly irreducible commutative rings, Duke Math. J. vol. 12 (1945) pp. 381-387.

22. G. C. Moisil, Sur les anneaux de caractéristique 2 ou 3 et leurs applications, Bulletin de Mathématiques et de Physique pures et Appliquées vol. 12 (1941) pp. 66-90.

23. S. Perlis, A characterization of the radical of an algebra, Bull. Amer. Math. Soc. vol. 48 (1942) pp. 128-132.

24. H. Prüfer, Neue Begriüdung der algebraischen Zahlentheorie, Math. Ann. vol. 94 (1925) pp. 198-243.

25. I. E. Segal, The group algebra of a locally compact group, Trans. Amer. Math. Soc. vol. 61 (1947) pp. 69-105.

26. M. H. Stone, The theory of representations for Boolean algebras, Trans. Amer. Math. Soc. vol. 40 (1936) pp. 37-111.

27. J. von Neumann, On regular rings. Proc. Nat. Acad. Sci. U. S. A. vol. 22 (1936) pp. 707-713.

28. - - Continuous geometry, Princeton University Lectures, 1936-1937 (Planographed).

29. L. I. Wade, Post algebras and rings, Duke Math. J. vol. 12 (1945) pp. 389-395.

\section{Smith College}

